\title{
Avaliação do estresse e das habilidades sociais na experiência acadêmica de estudantes de medicina de uma universidade do Rio de Janeiro
}

\author{
Eliane de Sousa Furtado \\ Eliane Mary de Oliveira Falcone \\ Cynthia Clark \\ Universidade do Estado do Rio de Janeiro
}

\begin{abstract}
Resumo
Estudos realizados em vários países indicam que estudantes de medicina estão expostos durante sua formação acadêmica a diversas situações geradoras de estresse. O despreparo do estudante para lidar com estas situações pode trazer repercussões importantes em seu desempenho acadêmico, em sua saúde e em seu bem-estar psicossocial. Além disso, pesquisas têm mostrado que deficiências em habilidades sociais podem contribuir para o desenvolvimento do estresse. Este estudo avaliou as fontes de estresse existentes no curso de medicina, assim como a relação entre os níveis de estresse e de habilidades sociais nos estudantes. A amostra constituiu-se de 178 alunos (105 mulheres e 73 homens) do primeiro ao sexto ano de medicina. O questionário para avaliação de estressores, o Inventário de Sintomas de Stress para Adultos de Lipp (ISSL) e o Inventário de Habilidades Sociais (IHS) revelaram 7 fontes de estresse mais apontadas pelos estudantes. Além disso, a maioria dos alunos encontra-se na fase de resistência ao estresse. Deficiências em habilidades sociais estão relacionadas ao estresse nesta população, sendo mais um fator somado às outras fontes estressoras. Conclui-se que a experiência acadêmica dessa amostra favorece o estresse e que níveis mais baixos de habilidades sociais estão relacionados à presença de estresse. Os dados obtidos neste estudo podem ser utilizados na elaboração de projetos de apoio psicológico para estudantes de medicina, bem como na melhoria do desenvolvimento acadêmico dos mesmos. Sugere-se que esta pesquisa seja replicada em outras faculdades de medicina para possibilitar uma comparação entre os resultados obtidos, os quais poderão confirmar, ampliar ou mesmo refutar os achados deste estudo.
\end{abstract}

Palavras-chave: Estresse em estudantes de medicina; habilidades sociais; estresse acadêmico.

\section{Abstract \\ Evaluation of stress and social skills in the academic experience of medical students of a Rio de Janeiro university}

Studies done in several countries have show that medical students are exposed to many situations that cause stress. The student's lack of preparation to deal with these situations can have important repercussions in regard to his/her performance, health and psychological and social well-being. Besides this, research has shown that deficiencies in social skills can contribute to the development of stress. This study evaluated sources of stress that exist within the medical course, as well as the relation between the levels of stress and social skills of the students. The sample consisted of 178 students (105 women and 73 men) from the first to the sixth level of medicine. The questionnaire to evaluate stressors, the Stress Symptom Inventory compiled by Lipp (ISSL) and Social Skills Inventory (IHS), revealed 7 sources of stress most quoted by the students. Besides this, the majority of the students are in a phase of resistance to stress. Deficiency in social skills is related to stress in this population and this is one more factor added to other sources of stress. It was concluded that the academic experience of this sample favors stress and to which low levels of social skills is related. The results obtained in this study can be used to elaborate projects which give psychological support to medical students and improvement to their academic development as well. It is suggested that this research be reproduced in other schools of Medicine so as to make possible a comparison between the obtained results, that will confirm, add or even oppose to the results of this study.

Keywords: stress in students of medicine; social skills; academic stress.

\section{O ESTRESSE NA EDUCAÇÃO MÉDICA}

A educação médica é uma experiência por vezes estressante, que pode ter um forte impacto em uma população jovem e potencialmente vulnerável. Embora se espere que os médicos sejam atenciosos, dedicados e empáticos com seus pacientes, a experiência profissionalizante nas faculdades tem, muitas vezes, colaborado para que os futuros médicos adquiram características contrárias às pretendidas (Cataldo Neto, Cavalet, Bruxel, Kappes \& Silva, 1998).

O termo estresse pode ser entendido como uma reação do organismo, com componentes físicos e/ou psicológicos, decorrente das alterações psicofisiológicas que ocorrem quando há um confronto com uma situação que provoque, de um 
modo ou de outro, irritação, medo, excitação ou confusão, ou mesmo que faça a pessoa imensamente feliz. Ele se mostra como um processo e não uma reação única, uma vez que um longo processo bioquímico instala-se quando a pessoa se depara com um estressor, cuja manifestação inicial ocorre de modo bastante semelhante, com o surgimento de taquicardia, sudorese excessiva, tensão muscular, boca seca e a sensação de estar alerta. Posteriormente, no desenvolvimento do processo do estresse, diferenças surgem de acordo com as predisposições genéticas do indivíduo, potencializadas pelo enfraquecimento desenvolvido ao longo da vida em função de acidentes ou doenças (Lipp, 1996).

É importante ressaltar, além do conceito de estresse, a definição de estressor. Tudo o que cause a quebra da homeostase interna, que exija alguma adaptação, pode ser chamado de um estressor. Fatos que envolvem adaptação a mudanças, sejam eles positivos ou negativos, constituem-se em estressores importantes porque a pessoa necessitará despender energia adaptativa para poder lidar com estes eventos (Lipp, 1996).

Os estressores podem ser externos e internos. Os primeiros são eventos externos ou condições externas que afetam o organismo. Eles ocorrem muitas vezes de modo independente do mundo interno da pessoa, como por exemplo: alteração de chefia, transformações políticas no país, acidentes e qualquer outra situação que aconteça fora do corpo e da mente da pessoa. Já os estressores internos são determinados completamente pelo próprio indivíduo, constituem-se do modo de ser da pessoa, se é ansiosa, tímida, depressiva ou se tem uma neurose. Outros estressores internos são: crenças irracionais, padrão tipo A de comportamento, falta de assertividade e dificuldade de expressão de sentimentos, entre outros (Lipp, 1996).

De acordo com Selye (1952, citado por Lipp, 1996), o processo de estresse desencadeia-se em três fases: alerta, resistência e exaustão. Apesar de Selye ter identificado apenas três fases do estresse, Lipp no decorrer da avaliação de seu instrumento (Inventário de Sintomas de Stress para Adultos de Lipp - ISSL) para diagnóstico do estresse identificou uma quarta fase. Esta nova fase, chamada de quase-exaustão, encontra-se entre a fase de resistência e a da exaustão e caracteriza-se pelo enfraquecimento da pessoa que não mais está conseguindo adaptar-se ou resistir ao estressor. Inicia-se o surgimento de doenças, no entanto, ainda não tão graves como na fase de exaustão. Mesmo apresentando desgaste e outros sintomas, a pessoa ainda é capaz de trabalhar e "funcionar" na sociedade até certo ponto, ao contrário do que ocorre na fase de exaustão, quando a pessoa pára de "funcionar" adequadamente, não conseguindo, na maioria das vezes, trabalhar ou concentrar-se (Lipp, 2000).

A resposta de estresse deve ser considerada em seus aspectos físicos e psicológicos. Na área emocional, o estresse pode produzir desde apatia, depressão, desânimo e sensação de desalento, hipersensibilidade emotiva, até raiva, ira, irritabilidade e ansiedade, além de ter o potencial de desencadear surtos psicóticos e crises neuróticas (Lipp, 1996).

Além de desencadear sintomas psicológicos, o estresse pode contribuir para a etiologia de várias doenças (hipertensão arterial essencial, úlceras gastroduodenais, câncer, psoríase, vitiligo) e afetar a qualidade de vida individual e de populações específicas (Lipp, 1996).

A respeito do papel do estresse na faculdade de medicina, estudos sugerem que o curso médico freqüientemente exerce efeitos negativos no desempenho acadêmico, na saúde e no bem-estar psicossocial do estudante.

Em um estudo acerca do estresse em estudantes de Medicina da Universidade Católica do Chile, Pallavicini, Venegas e Romo (1988) verificaram que $91 \%$ dos estudantes haviam experimentado um estado de tensão que variava de 3 a 4 dias até vários meses. A sintomatologia apresentada envolvia: tensão muscular, intranqüilidade, ansiedade, irritabilidade intensa, sintomas somáticos em diferentes órgãos, cansaço diante de esforços habituais ou esgotamento permanente acompanhados de desinteresse ou falta de concentração mental. Segundo os autores a freqüência dos sintomas somáticos aumentava do terceiro ao quinto ano, chegando a ser o fenômeno mais predominante neste último. Nesta pesquisa, os alunos mencionaram como principais causas de estresse as exigências acadêmicas excessivas e a falta de tempo para desenvolver suas aspirações afetivas e espirituais.

Outro estudo a respeito do estresse nesta população, realizado numa faculdade de medicina da Colômbia, mostrou que as principais fontes de estresse para esta população são: exames e provas, quantidade de material a ser estudado e sua dificuldade para ser aprendido, seguido de falta de tempo para familiares, amigos e lazer. Esta pesquisa aponta que o semestre de maior estresse é o terceiro e o de menor estresse é o primeiro (Restrepo, Jaramillo \& Marín, 1988).

Outro estudo sobre o tema, realizado na Faculdade de Medicina da Universidade de Alicante (Caruana, Martínez, Reig \& Merino, 1999), identificou cinco áreas principais de estresse em estudantes de medicina em ordem decrescente de importância: 1) sobrecarga acadêmica (exames); 2) relações e conflitos interpessoais; 3) problemas pessoais; 4) contato com a morte e o sofrimento; e 5) outros problemas relacionados com os estudos.

Um fator importante a considerar quando se analisa o estresse em estudantes de medicina é a presença de certas características inerentes ao aluno que podem torná-lo mais vulnerável ao estresse como, por exemplo, o perfeccionismo ou traços obsessivocompulsi-vos de personalidade.

Traços obsessivo-compulsivos de personalidade, que podem ter facilitado a admissão do aluno na escola médica, podem intensificar-se, tornando-se sintomas sob situações estressantes (Arnstein, 1986, citado por Miyasaki, 1997). 
O aluno com características perfeccionistas, quando acredita falhar ou não corresponder às expectativas nele depositadas, experimenta sentimentos tais como falta de controle, fracasso, desesperança, ansiedade, raiva e desespero, associados à depressão e ao suicídio (Blatt, 1995; Flett, Sawatzki \& Hewitt, 1995; Flett, Hewitt \& Singer, 1995; Flett, Hewitt \& De Rosa, 1996, citados por Miyasaki, 1997).

Segundo trabalhos de Millan, De Marco e Rossi (1990; 1992), alunos de medicina com melhor desempenho escolar encontram-se em um grupo de alto risco de suicídio. Os referidos autores conjeturam que, por serem pessoas mais exigentes, estariam mais propensas a sofrer as pressões impostas diante de qualquer falha. $\mathrm{O}$ estudante passa a ter culpa pelo que não sabe e com isso se sente paralisado pelo medo de errar. Esses quadros caracterizam-se por sentimentos de desvalia e impotência, que, muitas vezes, são responsáveis por idéias de abandono do curso, depressão e suicídio.

Apesar de ser mundialmente relatado em vasta bibliografia, o estresse na educação médica e suas possíveis conseqüências como somatizações, depressão e suicídio dentre outras, parece, por vezes, ser negligenciado no contexto educacional, tendo em vista que são poucas as universidades que contam com um serviço de apoio para os estudantes da área médica. Segundo Cataldo Neto e cols. (1998), as escolas médicas precisam aprimorar o padrão de ensino que ministram, adaptando-se e interagindo melhor com o desenvolvimento de seu objeto de ensino: o estudante de medicina, com suas características psicossociais e com o estresse ao qual é submetido.

Embora possa ser difícil modificar as condições do curso de medicina que proporcionam estresse ao estudante, a implementação de programas para o desenvolvimento de repertórios comportamentais adequados ou treino em solução de problemas pode auxiliar estudantes a lidarem com as dificuldades presentes na vida acadêmica (Bligh, 1995; Ingenito \& Wooles, 1995; Meichenbaum, 1987; Smith, Lyles, Mettler \& Marshall, 1995, citados por Miyasaki, 1997).

\section{HABILIDADES SOCIAIS}

As rápidas transformações pelas quais tem passado a sociedade, principalmente a partir do século XX, têm demandado das pessoas não só o domínio de habilidades técnicas, mas também um desempenho socialmente competente para que se tenham relações profissionais e sociais mais satisfatórias, duradouras e gratificantes.

A respeito do conceito de habilidades sociais não existe ainda um consenso na literatura. As habilidades sociais têm sido consideradas por alguns autores como sinônimo de assertividade (Caballo, 1991, 1993, 1995). No entanto, outros autores apontam que a habilidade assertiva não esgota a noção de habilidades sociais (Del Prette \& Del Prette, 1999; Falcone, 1989, 1995, 1998; MacKay, 1988; Matos, 1997). Uma revisão de estudos feita por Falcone $(1998,2000)$ sugere fortemente que a empatia também é uma habilidade social e que, em alguns contextos, é mais apropriada do que a assertividade. Além disso, a assertividade e a empatia se complementam em várias situações sociais. A capacidade para solucionar problemas interpessoais também tem sido apontada como necessária para a manutenção da qualidade das relações interpessoais.

Bedell e Lennox (1997) propõem que o indivíduo socialmente habilidoso é capaz de obter ganhos com maior freqüência, desempenhar o mínimo possível de tarefas indesejáveis, além de desenvolver e manter relacionamentos mutuamente benéficos e sustentadores. Assim, indivíduos socialmente habilidosos buscam constantemente a satisfação pessoal, sem, contudo descuidar da qualidade de suas interações.

As habilidades sociais têm sido associadas a melhor saúde física e mental, ao sucesso profissional e a maior realização pessoal (Caballo, 1987, 1991; Collins \& Collins, 1992; Goleman, 1995; Ickes, 1997). Para Rubio e Anzano (1998) as habilidades sociais se mostram como recursos indispensáveis para realizar atividades fundamentais da vida como nos relacionamentos interpessoais, na construção de amizades e desenvolvimento de redes sociais, no trabalho em equipe, na prestação de serviços profissionais, dentre outros.

Se por um lado, estes aspectos a respeito das habilidades sociais revelam a importância destas para a qualidade de vida, a saúde, além da satisfação pessoal e profissional, por outro lado, Del Prette e Del Prette (2001, p. 7) destacam estudos que revelam deficiências em habilidades sociais como geralmente relacionadas "a uma pior qualidade de vida e a diversos tipos de transtornos psicológicos como a timidez, o isolamento social, a delinqüência juvenil, o desajustamento escolar, o suicídio e os problemas conjugais, além de outras síndromes como a depressão, o pânico social e a esquizofrenia".

As deficiências em habilidades sociais atingem cerca de 25 a $30 \%$ dos pacientes com transtornos emocionais (Argyle, 1984). Deficiências em habilidades sociais estão presentes também em neuroses histéricas, uso de drogas e alcoolismo, observando-se nestes últimos, dificuldades interpessoais que facilitam a ingestão de álcool ou consumo de substâncias (Hidalgo \& Abarca, 2000).

Estudos têm mostrado que a falta de destrezas sociais repercute nas pessoas de maneiras diferentes. Algumas vezes apresentam um desempenho acadêmico inferior ao das pessoas com habilidades adequadas, outras, inibição de criatividade. A vida familiar e conjugal pode ser afetada especialmente em termos de comunicação afetiva, expressão de sentimentos positivos e negativos, resolução de conflitos e negociação (Hidalgo \& Abarca, 2000).

Outro ponto importante a ser ressaltado é que deficiências em habilidades sociais, tais como a falta de assertividade e a dificuldade de expressão dos sentimentos, têm sido relacionadas ao estresse. A inabilidade social tem sido apontada na literatura 
como um estressor interno, ou seja, relacionado à forma como os indivíduos pensam e comportam-se (Lipp \& Rocha, 1994, citados por Lipp, 1996). De acordo com Ayres (1996), muitos estudos têm constatado que fontes internas de estresse tais como, padrão de comportamento tipo $\mathrm{A}$ e falta de assertividade, estão muitas vezes presentes em pessoas hipertensas. Pesquisas realizadas no Brasil e nos Estados Unidos mostram que muitos hipertensos apresentam dificuldades em defender seus direitos e expressar seus sentimentos, o que se traduz em uma aparente inabilidade social.

Déficits em habilidades sociais também têm sido relacionados ao estresse em atletas. Uma revisão de estudos feita por Maciel (1996) aponta a existência de baixa assertividade em ambientes esportivos afetando o desempenho dos atletas. A comunicação eficiente é um elemento crucial para o sucesso atlético, sendo que falhas de comunicação são comumente encontradas no trabalho com grupos esportivos. Além disso, muitos atletas nunca foram ensinados ou reforçados para as habilidades de comunicação básica, já que em seus históricos de vida podem não ter sido enfatizadas as habilidades assertivas. Baixos níveis de assertividade podem estar associados à falta de confiança, uma vez que atletas não confiantes são geralmente mais facilmente intimidados por pares ou por figuras de autoridade e podem ser menos seguros de suas convicções ao manifestar suas opiniões. O treinamento da assertividade pode ter um importante papel na prevenção e no trato dos problemas de comunicação comumente encontrados em ambientes esportivos, que podem estar contribuindo para a existência do estresse nestes mesmos ambientes.

Até agora vimos que estudos em muitos países mostram que a formação médica, pela sua própria natureza, viabiliza experiências de estresse ao estudante de medicina, em especial aos mais vulneráveis e que deficiências em habilidades sociais podem contribuir para o desenvolvimento do estresse. Estas evidências despertaram o interesse para a realização desta pesquisa que visa identificar se os alunos de medicina da UNIRIO vivenciam situações estressantes e se o nível de habilidades sociais destes estudantes está relacionado à ocorrência de estresse nesta população. Neste sentido, o presente estudo tem como objetivos: 1) identificar as principais fontes de estresse na amostra total de alunos de medicina estudada; 2) identificar se existem diferenças entre os sub-grupos (anos do curso e sexo) estudados a respeito das principais fontes de estresse; 3) avaliar se os estudantes apresentam estresse e em qual(is) fase(s) de estresse se encontram, bem como se existem diferenças entre os sub-grupos (anos do curso); 4) avaliar a existência de relação entre o nível de estresse e de habilidades sociais.

Tendo em vista que o estudante muitas vezes encontra-se despreparado para lidar com as situações estressantes e que isso pode trazer repercussões importantes na sua vida pessoal e acadêmica, este trabalho torna-se útil para o aprofundamento deste tema, uma vez que existem poucas pesquisas em nosso país sobre o assunto. Neste sentido, os dados desta pesquisa podem ser de grande utilidade para elaboração de projetos de apoio psicológico ao estudante, bem como para a melhoria do desenvolvimento acadêmico desses estudantes e podem ainda servir de base para futuras pesquisas.

\section{MÉTODO}

\section{1) Material}

Para a obtenção de dados foram utilizados os seguintes instrumentos:

A) Ficha para obtenção de dados demográficos. Utilizada para caracterizar a amostra em termos de sexo, idade, estado civil, ano de faculdade, reprovações e profissão;

B) Questionário para avaliação de estressores em estudantes de medicina. Construído a partir da adaptação do Inventário de Situações de Estresse para Estudantes de Medicina (Caruana, Reig \& Merino, 1991) e do Questionário para Identificação de Fontes de Estresse em Estudantes de Medicina (Restrepo \& cols., 1988). Constitui-se de 53 fontes potencialmente estressoras a serem avaliadas pelos estudantes em termos de intensidade, dispondo estes de 5 alternativas de resposta: 1) Não me produz estresse. 2) Produz estresse leve. 3) Produz estresse moderado. 4) Produz bastante estresse. 5) Produz muitíssimo estresse. Além disso, o estudante deve assinalar os estressores que estiverem ocorrendo com ele no momento. A pontuação total mínima do questionário é de 53 e a máxima de 265. As 53 fontes potencialmente estressoras estão relacionadas a aspectos da vida dos estudantes tais como: estudos (20 itens), relações com os professores e a Universidade (3 itens), pacientes (9 itens), família (2 itens), relacionamento interpessoal (5 itens), saúde física e psíquica (2 itens), questões pessoais (10 itens), estressores relacionados a outros aspectos (2 itens);

C) Inventário de Sintomas de Stress para Adultos de Lipp (ISSL, 2000);

D) Inventário de Habilidades Sociais (IHS - Del Prette \& Del Prette, 2001).

\section{1) Participantes}

Participaram desta pesquisa 178 estudantes do primeiro ao sexto ano do curso de medicina de uma universidade pública do Rio de Janeiro, sendo 29 do primeiro ano, 41 do segundo, 35 do terceiro, 22 do quarto, 32 do quinto e 19 do sexto. Quanto ao gênero 105 estudantes são do sexo feminino e 73 do sexo masculino, com idades variando entre 17 e 41 (média $=22,15$ anos $)$.

\section{2) Procedimento}


Os questionários foram aplicados em sala de aula nos alunos do segundo, terceiro e quinto anos. Os alunos do primeiro, quarto e sexto anos levaram os questionários para casa com posterior devolução. Os alunos foram orientados a responder individualmente aos questionários.

\section{3) Tratamento dos dados}

Os dados foram analisados através do programa estatístico S.P.S.S. 8.0 para Windows. Utilizou-se estatística descritiva (média, desvio padrão e porcentagem), bem como os testes estatísticos t de Student e Qui-Quadrado considerando o nível de significância de $5 \%(\mathrm{p}<0,05)$.

\section{RESULTADOS}

As características da amostra indicam que a maior parte dos estudantes é solteira $(98,3 \%)$. A respeito de reprovações durante o curso, 14 alunos $(7,9 \%)$ relataram terem repetido um período. A maioria dos estudantes não trabalha $(82,9 \%)$ e dentre aqueles que trabalham a maior parte está envolvida com atividades ligadas ao curso, tais como: estágios, plantões e monitoria. A seguir serão apresentados os dados obtidos a partir dos instrumentos utilizados no estudo.

\section{Avaliação dos estressores}

O questionário para avaliação de estressores em estudantes de medicina indicou que os professores injustos (média=3,94; $\mathrm{sd}=1,17$ ), a excessiva quantidade de matéria para estudo (média=3,93; $\mathrm{sd}=1,06$ ), a grande quantidade de provas (média $=3,80$; $\mathrm{sd}=1,00$ ), as provas orais (média $=3,74 ; \mathrm{sd}=1,28$ ), a falta de tempo para diversão (média $=3,61 ; s d=1,19$ ), as expectativas como futuro médico (média $=3,49$; $\mathrm{sd}=1,27)$ e o medo de fracassar nos estudos (média $=3,48 ; \mathrm{sd}=1,34$ ), foram considerados pelos estudantes, em ordem decrescente, os estressores mais intensos.

Dentre os estressores apontados como mais intensos alguns se apresentam comuns a todos os cursos, tais como: os professores injustos e a excessiva quantidade de matéria para estudo. Os estressores: grande quantidade de provas, falta de tempo para diversão e provas orais, mostraram-se comuns a todos os anos de curso, excluindo-se o sexto. $\mathrm{O}$ estressor referente às expectativas como futuro médico aparece em todos os anos do curso, com exceção do primeiro e segundo. $\mathrm{O}$ estressor relacionado a provas de residência aparece no quinto e sexto anos.

Quanto ao gênero, observaram-se algumas diferenças a respeito dos estressores mais intensos. Para as mulheres, os estressores mais apontados foram em ordem decrescente: excessiva quantidade de matéria para estudo, os professores injustos, grande quantidade de provas, provas orais, pouco tempo para dormir, medo de fracassar nos estudos e medo de cometer um erro no diagnóstico ou no tratamento. Já para os homens, as fontes de estresse mais intensas foram: os professores injustos, falta de tempo para diversão, excessiva quantidade de matéria para estudo, grande quantidade de provas, provas orais, provas de residência médica e expectativas como futuro médico.

Com relação à pontuação total média obtida no questionário para avaliação de estressores pela amostra global, obteve-se o valor de 133,98. Utilizando-se o teste t de Student foram encontradas diferenças significativas $(\mathrm{p}=0,000)$ entre as médias quanto ao gênero. As mulheres apresentaram uma pontuação mais alta (média=141,29; sd=26,93) que a dos homens (média=123,48; sd=29,81), sugerindo, portanto, que elas são mais suscetíveis às fontes de estresse, percebendo-as de forma mais intensa.

\section{Avaliação do estresse}

Com relação ao nível de estresse, o Inventário de Sintomas de Stress para Adultos de Lipp (ISSL) mostrou que $65,2 \%$ dos alunos têm estresse. Dentre estes, a maioria $(60,7 \%)$ encontra-se na fase de resistência, enquanto 3,9\% estão na fase de quase exaustão e apenas $0,6 \%$ na fase de alerta. $\mathrm{O}$ estresse está ausente em $34,8 \%$ dos alunos.

Os anos do curso que apresentaram maior percentual de estudantes com estresse, de acordo com o ISSL, foram respectivamente o primeiro $(93,1 \%)$, o segundo $(85,4 \%)$ e o sexto $(68,5 \%)$.

Ao comparar-se as pontuações médias obtidas no questionário para avaliação de estressores pelos grupos de alunos sem estresse e com estresse na fase de resistência, utilizando-se o teste $\mathrm{t}$ de Student, observou-se diferença significativa entre estas $(\mathrm{p}=0,000)$. A pontuação mais alta apresentada pelos alunos com estresse (média $=143,48 ; \quad s d=25,89$ ) quando comparada a dos alunos sem estresse (média=116,40; $\mathrm{sd}=26,23$ ) indica que os primeiros são afetados de forma mais intensa pelos estressores.

\section{Habilidades Sociais X Estresse}

$\mathrm{O}$ teste estatístico Qui-quadrado não apresentou relação entre os níveis de estresse (obtidos pelo ISSL) e de habilidades sociais (obtidos pelo IHS) na população total, $p=0,164$. No entanto, levando-se em consideração o sexo, observou-se uma relação significativa para o grupo dos homens $(\mathrm{p}=0,008)$, conforme indicado na tabela. Neste grupo, a maior parte dos sujeitos com repertório elaborado de habilidades sociais (HS) não apresentou estresse, enquanto sujeitos com déficits em HS em sua maioria apresentaram estresse. No grupo das mulheres, a maior parte daquelas que tinham déficits em HS apresentaram estresse, tendo, portanto, um resultado semelhante ao dos homens. No entanto, a maioria das mulheres com repertório elaborado de HS também apresentaram estresse, sendo este resultado diferente ao dos homens.Tabela 1 - Relação entre níveis de estresse e habilidades sociais por gênero

\begin{tabular}{ccc}
\hline Sem & Fase de & Total \\
Estresse & $\begin{array}{c}\text { Resistência } \\
\text { Resten }\end{array}$ & \\
\hline
\end{tabular}




\begin{tabular}{|c|c|c|c|c|}
\hline \multirow{4}{*}{ 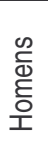 } & Baixa HS & 12 & 20 & 32 \\
\hline & $\%$ & 32,5 & 62,5 & 100 \\
\hline & Alta HS & 17 & 6 & 23 \\
\hline & $\%$ & 73,9 & 26,1 & 100 \\
\hline \multirow{4}{*}{$\begin{array}{l}\mathscr{W} \\
\frac{\Phi}{\Phi} \\
\stackrel{\bar{J}}{\Sigma}\end{array}$} & Baixa HS & 6 & 13 & 19 \\
\hline & $\%$ & 31,6 & 68,4 & 100 \\
\hline & Alta HS & 6 & 20 & 26 \\
\hline & $\%$ & 23,1 & 76,9 & 100 \\
\hline
\end{tabular}

Homens $(p=0,008)$ Mulheres $(p=0,381)$

\section{DISCUSSÃO}

\section{Avaliação dos estressores}

As dificuldades presentes na formação médica e o impacto que as mesmas podem exercer na vida acadêmica e no bem-estar físico e psicossocial do estudante têm sido objeto de estudo de pesquisas em vários países.

O presente estudo pôde demonstrar que os alunos do curso de medicina de uma das mais importantes faculdades do Estado do Rio de Janeiro também estão em contato com uma série de dificuldades que se tornam, para muitos, verdadeiras fontes de estresse.

Esta pesquisa confirma estudos anteriores a respeito dos estressores mais intensos apontados pelos alunos: professores injustos, a excessiva quantidade de matéria para estudo, a grande quantidade de provas, as provas orais, a falta de tempo para diversão, as expectativas como futuro médico e o medo de fracassar nos estudos (Caruana \& cols., 1999; Pallavicini \& cols., 1988; Restrepo \& cols., 1988).

A partir das fontes de estresse mais apontadas, pode-se dizer que as maiores preocupações dos estudantes estão relacionadas à área acadêmica no que diz respeito à sobrecarga, exigências e desempenho, e que estas preocupações estão presentes ao longo de todo o curso de medicina. Neste sentido, é importante destacar peculiaridades presentes em alguns anos do curso. O estressor: expectativas como futuro médico, não aparece na lista dos mais intensos nos primeiros anos de curso (pré-clínicos) e passa a ser uma preocupação a partir do terceiro ano, quando se inicia o contato com o paciente. No sexto ano, quando começa o internato, os alunos não têm mais aulas em sala e nem provas; provavelmente por esta razão os estressores: grande quantidade de provas e provas orais, não aparecem no sexto ano como os mais intensos. $\mathrm{Na}$ fase final do curso surgem outras preocupações, tais como as disputadas provas de residência médica que exigem uma grande dedicação por parte do aluno.

A análise da pontuação total obtida no questionário para avaliação dos estressores, levando em consideração o gênero, mostrou que as mulheres percebem os estressores de forma mais intensa que os homens. Tais resultados confirmam estudos anteriores que indicam serem as mulheres mais suscetíveis às fontes de estresse (Caruana \& cols., 1999).

\section{Avaliação do estresse}

Os resultados obtidos através do ISSL mostraram que a maioria dos estudantes apresenta estresse, confirmando vários estudos que indicam ser a educação médica uma experiência estressante para os alunos (Cataldo Neto \& cols., 1998; Nader \& Barros, 1991; Pallavicini \& cols., 1988; Restrepo \& cols., 1988; Caruana \& cols., 1999). A fase de estresse predominante nesta população é a de resistência. Além disso, o estresse permanece presente durante todo o curso, mas em diferentes níveis para cada ano, estando as maiores incidências nos primeiros anos (confirmando o estudo de Pólo, Hernandez \& Pozo, 1996, citado por Miyasaki, 1997) e no final do curso. 
Uma possibilidade para a alta ocorrência de estresse nos primeiros anos de curso é o fato do ingresso na faculdade ser uma fase de transição em termos de estilo de vida, exigindo, portanto, um período de adaptação às diversas mudanças inerentes à vida acadêmica. $\mathrm{O}$ aumento da ocorrência de estresse no último ano pode estar associado ao exame de residência, que corresponde a um novo vestibular para muitos alunos.

A prevalência do estresse na fase de resistência nos alunos de medicina indica que os mesmos conseguem resguardar-se até certo ponto dos estímulos estressores, uma vez que apenas 3,9\% encontravam-se na fase de quase-exaustão e nenhum chegou à fase de exaustão. Apesar de poucos alunos terem atingido fases mais graves de estresse, é importante mencionar que a fase de resistência do estresse se caracteriza pela busca da homeostase pelo organismo quando este se vê diante de um estressor permanentemente atuante, ocorrendo então uma contínua hiperatividade córtico supra-renal e um gasto de energia excessiva e necessária para outras funções do corpo (Selye, 1965, citado por Lipp, 1996). Também segundo Lipp (1987) a contínua presença do estresse pode afetar o sistema imunológico tornando o organismo vulnerável a várias infecções e doenças.

Outro ponto importante foi a maior média obtida no Questionário para Avaliação de Estressores pelos alunos com estresse na fase de resistência, quando comparados aos alunos sem estresse. Estes resultados indicam que os alunos com estresse vêem as situações presentes na vida acadêmica como mais estressantes, e que possivelmente apresentam maior dificuldade para lidar com as mesmas.

\section{Habilidades Sociais X Estresse}

A análise da relação entre habilidades sociais (HS) e estresse mostrou que deficiências em HS estão associadas à ocorrência de estresse na amostra estudada. Tal resultado vem corroborar a concepção bastante difundida na literatura de que déficits em HS estão geralmente relacionados a uma série de problemas que incluem: estresse (Ayres, 1996; Lipp \& Rocha, 1994; Maciel, 1996), insegurança, baixa autoestima, ansiedade, pior qualidade de vida, transtornos psicológicos, dentre outros (Argyle, 1984; Del Prette \& Del Prette, 2001; Hidalgo \& Abarca, 2000; Rubio \& Anzano, 1998). Parece que as deficiências em habilidades sociais nos estudantes de medicina são mais um fator na produção de estresse que vêm somar-se às outras fontes estressoras presentes na vida acadêmica.

Também é interessante observar que deficiências em habilidades sociais representaram uma maior incidência de estresse tanto em homens quanto em mulheres. No entanto, a existência de altas habilidades sociais mostrou-se associada a uma baixa incidência de estresse apenas para os homens, por este motivo, encontrou-se relação significativa entre habilidades sociais (altas e baixas) e estresse apenas na amostra masculina. Na população feminina, pelo contrário, parece que ter um alto nível de habilidades sociais contribui para uma maior ocorrência de estresse.
É importante ressaltar que as habilidades sociais avaliadas através do instrumento utilizado neste estudo enfatizam, em sua maioria, habilidades ligadas à assertividade. Neste sentido, pode-se dizer que a alta assertividade na população feminina está relacionada à presença de estresse na mesma. Talvez este dado possa ser explicado por estudos que evidenciam que o gênero da pessoa assertiva é um fator de influência na maneira como o comportamento assertivo será percebido (Broverman I., Broverman D., Clarkson, Rosenkrantz \& Vogel, 1970; Hess, Bridgwater, Bornstein \& Sweeney, 1980; Jakubowski-Spector, 1973; Kelly \& Worell, 1977; Spence \& Helmreich, 1972, citados por Kelly, Kern, Kirkley \& Patterson, 1980). Pesquisas mostram que o comportamento assertivo exibido por mulheres resulta em avaliações sociais mais negativas que o comportamento idêntico em homens (Cowan \& Koziej, 1979, citados por Kelly \& cols., 1980).

Este tipo de avaliação diferencial parece ser uma consequiência do estereótipo conservador a respeito dos papéis sexuais femininos, que tem historicamente desvalorizado o comportamento assertivo em mulheres e, no entanto, reforça este comportamento em homens (Kelly \& cols., 1980; Kern, Cavell \& Beck, 1985). Os estudos de Kelly e cols. (1980) e de Kern e cols. (1985) indicam que pessoas possuidoras de uma atitude conservadora para os papéis de mulheres em sociedade percebem o comportamento assertivo feminino como menos agradável, amigável, compreensivo, competente, habilidoso e atrativo.

Levando em consideração estas afirmações a respeito do comportamento assertivo feminino, é possível levantar a idéia de que as alunas deste estudo com altas habilidades assertivas tenham recebido respostas negativas ou que tenham sido até mesmo punidas no ambiente da faculdade de medicina.

As reações sociais negativas para o comportamento assertivo podem ter contribuído fortemente para a alta incidência de estresse nas mulheres com altas habilidades sociais. Além disso, tais reações negativas podem levar a uma inibição de comportamentos assertivos por parte destas mulheres. A inibição da assertividade, por sua vez, pode contribuir para o surgimento de estresse, já que quando uma pessoa costuma se comportar assertivamente ela facilita a solução de problemas interpessoais, aumenta o senso de auto-eficácia e a auto-estima, melhora a qualidade dos relacionamentos e sente-se mais tranquiila (Caballo, 1991).

Sugere-se que novos estudos sejam realizados para um melhor esclarecimento a respeito das diferenças nos resultados entre homens e mulheres quanto à alta habilidade social. Seria interessante que as próximas pesquisas utilizassem instrumentos que avaliassem os outros aspectos das habilidades sociais, tais como, empatia e solução de problemas.

Os resultados obtidos através deste estudo podem ser de grande utilidade para aqueles que se interessam pelo estresse na faculdade de medicina, bem como para os que estudam a importância das habilidades sociais, já que em nosso país existem poucas pesquisas sobre estes temas e mais especificamente sobre esta população. Apesar de existirem alguns 
estudos nacionais a respeito do estresse provocado pela educação médica, esta pesquisa parece ser pioneira quanto aos instrumentos utilizados, bem como pelo estudo das habilidades sociais em alunos de medicina. Deste modo, seria interessante que esta pesquisa fosse replicada em outras faculdades de medicina para possibilitar uma comparação entre os resultados obtidos, os quais poderão confirmar, ampliar ou mesmo refutar os achados deste estudo.

Os estudantes de medicina "sobrevivem" aos estressores. No entanto, é necessário reconhecer que eles existem e que podem prejudicar o funcionamento psicossocial do aluno, bem como de seu futuro desempenho profissional. Neste sentido, torna-se importante o desenvolvimento de serviços de assistência psicológica aos estudantes de medicina que visem não apenas o tratamento clínico de distúrbios psicológicos, mas também o oferecimento de programas de desenvolvimento de habilidades para lidar com os estressores. Estes programas podem auxiliar o aluno a identificar os fatores de risco para o surgimento de estresse, a reconhecer em si a ocorrência de eventuais dificuldades e a utilizar estratégias adequadas para lidar com elas.

Os momentos nos quais os programas de auxílio ao estudante parecem mais necessários são no início e no final do curso. Tais programas podem incluir treino de controle do estresse, bem como treinamento em habilidades sociais (assertividade, empatia e solução de problemas). Os resultados do presente estudo podem contribuir para a implementação destes programas em faculdades de medicina.

\section{REFERÊNCIAS}

Argyle, M. (1984). Some new developments in social skills training. Bulletin of the British Psychological Society, 53, 405410.

Arnstein, R. L. (1986). Emotional problems in medical students. American Journal of Psychiatry, 143, 1422-1423.

Ayres, A. M. M. (1996). Stress e afetividade nos hipertensos. Em M. Lipp (Org.), Pesquisas sobre stress no Brasil: Saúde, ocupações e grupos de risco (pp. 71-81). São Paulo: Papirus.

Bedell, J. R. \& Lennox, S. S. (1997). Handbook for communication and problem-solving skills training: A cognitive-behavioral approach. New York: Wiley.

Blatt, S. J. (1995). The destructiveness of perfectionism: Implications for the treatment of depression. American Psychologist, 50, 1003-1020.

Bligh, J. (1995). Problem-based learning in medicine: An introduction. Postgraduate Medical Journal, 71, 323-326.

Broverman, I. K.; Broverman, D.; Clarkson, E.; Rosenkrantz, P. \& Vogel. (1970). Sex role stereotypes and clinical judgments of mental health. Journal of Consulting and Clinical Psychology, $34,1-7$.

Caballo, V. E. (1987). Teoría, evaluación y entrenamiento de las habilidades sociales. Valencia: Promolibro.

Caballo, V. E. (1991). El entrenamiento en habilidades sociales. Em V. E. Caballo (Org.), Manual de técnicas de terapia y modificación de conducta (pp. 403-471). Madrid: Siglo Veintiuno.

Caballo, V. E. (1993). Manual de evaluación y entrenamiento de las habilidades sociales. Madrid: Siglo veintiuno.

Caballo, V. E. (1995). Una aportación española a los aspectos moleculares, a la evaluación y al entrenamiento de las habilidades sociales. Revista Mexicana de Psicologia, 12, 121131 .
Caruana, A.; Reig, A. \& Merino, J. (1991). Bateria de evaluacion en estudiantes de medicina. Mimeo. Departamento de Medicina y Psiquiatría. Universidad de Alicante.

Caruana, A.; Martínez, E. R.; Reig, A. \& Merino, J. (1999). Evaluación del estrés en estudiantes de medicina. Ansiedad y estrés, 5 (1), 79-97.

Cataldo Neto, A.; Cavalet, D.; Bruxel, D. M.; Kappes, D. S. \& Silva, D. O. F. (1998). O estudante de medicina e o estresse acadêmico. Revista de Medicina da PUCRS, 8 (1), 6-12.

Collins, J. \& Collins, M. (1992). Social skills training and the professional helper. New York: Willey.

Cowan, G. \& Koziej, J. (1979). The perception of sex inconsistent behavior. Sex Roles, 1, 1-10.

De Marco, O. L. N.; Rossi, E. \& Millan, L. R. (1992). Considerações acerca do "erro médico" e de suas implicações psicológicas. Revista ABP-APAL, 14 (2), 67-70.

Del Prette, Z. A. \& Del Prette, A. (1999). Psicologia das habilidades sociais. Petrópolis: Editora Vozes.

Del Prette, Z. A. P. \& Del Prette, A. (2001). Inventário de habilidades sociais: Manual de aplicação, apuração $e$ interpretação. São Paulo: Casa do Psicólogo.

Falcone, E. O. (1989). A eficácia do tratamento em grupo da ansiedade social. Psicologia Clínica, Pós-Graduação e Pesquisa PUC-RJ, 4, 75-91.

Falcone, E. O. (1995). Grupos. Em B. Rangé (Org.), Psicoterapia comportamental e cognitiva: Pesquisa, prática, aplicações e problemas (pp. 159-169). Campinas: Editorial Psy.

Falcone, E. O. (1998). A avaliação de um programa de treinamento da empatia. Dissertação de Doutorado, Curso de Pós-Graduação em Psicologia Clínica, Universidade de São Paulo, São Paulo.

Falcone, E. (2000). Habilidades sociais: Para além da assertividade. Em R. C. Wielenska (Org.), Sobre comportamento e cognição: Questionando e ampliando a teoria e as intervenções clínicas e em outros contextos (pp. 211-221). Vol 6. São Paulo: Set Editora Ltda.

Flett, G. L.; Hewitt, P. L. \& Singer, A. (1995). Perfectionism and parental authority styles. Individual Psychology. Journal of Adlerian Theory, Researsh \& Practice, 51, 50-60.

Flett, G. L.; Sawatzki, D. L. \& Hewitt, P. L. (1995). Dimensions of perfectionism and goal commitment: A further comparison of two perfectionism measures. Journal of Psychopathology and Behavioral Assessment, 17, 111-124.

Flett, G. L.; Hewitt, P. L. \& De Rosa, T. (1996). Dimensions of perfectionism, psychosocial adjustment, and social skills. Personality \& Individual Differences, 20, 143-150.

Goleman, D. (1995). Inteligência emocional. Rio de Janeiro: Objetiva.

Hess, E. P.; Bridgwater, C. A.; Bornstein, P. H. \& Sweeney, T. M. (1980). Situational determinants in the perception of assertiveness: Gender-related influences. Behavior Therapy, $11,49-58$

Hidalgo, C. G. C. \& Abarca N. M. (2000). Comunicacion interpessoal: Programa de entrenamiento en habilidades sociales $\left(5^{2}\right.$ ed.). Santiago de Chile: Ediciones Universidad Católica de Chile.

Ickes, W. (1997). Introducion. Em W. Ickes (Org.), Empathic accuracy (pp.1-16). New York: Guilford.

Ingenito, A. J. \& Wooles, W. R. (1995). Survey results of POPS (Pacient-Oriented-Problem-Solving) use in United States and Canadian schools of medicine and pharmacy. Journal of Clinical Pharmacology, 35, 117-127.

Jakubowski-Spector, P. (1973). Facilitating the growth of women through assertive training. The Counseling Psychologist, 4, $75-86$

Kelly, J. A. \& Worell, J. (1977). New formulations of sex roles and androgyny: A critical review. Journal of Consulting and Clinical Psychology, 45, 1101-1115.

Kelly, J. A.; Kern, J. M.; Kirkley, B. G. \& Patterson, J. N. (1980). Reactions to assertive versus unassertive behavior: Differential effects for males and females and implications for assertiveness training. Behavior Therapy, 11, 670-682.

Kern, J. M.; Cavell, T. A. \& Beck, B. (1985). Predicting differential reactions to males' versus females' assertions, 
empathic-assertions, and nonassertions. Behavior Therapy, 16, 63-75.

Lipp, M. (1987). Como enfrentar o stress. São Paulo: Ícone.

Lipp, M. \& Rocha, J. C. (1994). Stress, hipertensão e qualidade de vida. Campinas: Papirus.

Lipp, M. (1996). Stress: Conceitos básicos. Em M. Lipp (Org.), Pesquisas sobre stress no Brasil: Saúde, ocupações e grupos de risco (pp. 17-31). São Paulo: Papirus.

Lipp, M. (2000). Manual do Inventário de Sintomas de Stress para Adultos de Lipp. São Paulo: Casa do Psicólogo.

Maciel, S. V. (1996). Atleta Juvenil Feminina: Correlação entre características psicológicas, stress e lesões osteomusculares. Em M. Lipp (Org.), Pesquisas sobre stress no Brasil: Saúde, ocupações e grupos de risco (pp. 211-224). São Paulo: Papirus.

MacKay, D. (1988). Dificuldades sociais e interpessoais. Em: H. Lettner e B. Rangé (Orgs.), Manual de psicoterapia comportamental (pp. 137-148). São Paulo: Manole.

Matos, M. G. (1997). Comunicação e gestão de conflitos na escola. Lisboa: Edições FMH.

Meichenbaum, D. (1987). Manual de inoculación de estrés. Barcelona: Martínez-Roca.

Millan, L. R.; Rossi, E. \& De Marco, O. L. N. (1990). O suicídio entre estudantes de medicina. Revista do Hospital das Clínicas São Paulo, 45, 145-149.

Miyazaki, M. C. O. S. (1997). Psicologia na formação médica: Subsídios para prevenção e trabalho clínico com universitários. Dissertação de Doutorado, Curso de Pós-Graduação em Psicologia Clínica, Universidade de São Paulo, São Paulo.

Nader, D. A. \& Barros, A. F. (1991). Estudo de perturbações psicossomáticas em estudantes de medicina da UFJF. H.U. Revista Juiz de Fora, 18 (1), 69-79.
Pallavicini, J. G.; Venegas, L. R. \& Romo, O. V. (1988). Estrés en estudiantes de medicina de la Universidad Catolica de Chile. Revista de Psiquiatría Clínica Santiago de Chile, 25 (1), 23 29.

Polo, A.; Hernandez, J. M. \& Pozo, C. (1996). Evaluación del estrés académico en estudiantes universitarios. Ansiedad y Estrés, 2, 159-172.

Restrepo, A. R.; Jaramillo, F. R. \& Marín, J. C. R. (1988). Estrés en estudiantes de medicina del Instituto de Ciencias de La Salud. Revista CES Medicina Colombia, 2 (1), 38-43.

Rubio, J. M. L. \& Anzano, S. M. (1998). Aproximación conceptual a las habilidades sociales. Em F. Gil \& J. M. L. Rubio (Orgs.), Habilidades sociales: Teoría, investigación e intervención (pp. 13-23). Madrid: Editorial Sintesis.

Selye, H. A. (1952). The story of the adaptacionsyndrome. Montreal, Acta.

Selye, H. A. (1965). Stress: A tensão da vida (2a ed.). (F. Branco, Trad.). São Paulo: Ibrasa.

Smith, R. C.; Lyles, J. S.; Mettler, J. A. \& Marshall, A. A. (1995). A strategy for improving patient satisfaction by the intensive training of residents in psychosocial medicine: A controled, randomized study. Academic Medicine, 70, 729-732.

Spence, J. T. \& Helmreich, R. (1972). Who likes competent women? Competence, sex-role congruence of interest and subjects' attitudes toward women as determinants of interpersonal attraction. Journal of Applied Social Psychology, 2, 197-213.

Recebido: 10.10 .2003

Revisado: $1^{\circ} .12 .2003$

Aceito: 05.12.2003

\section{Nota:}

${ }^{1}$ Agradecemos aos professores e alunos da Faculdade de Medicina da UNI-RIO pela disponibilidade em colaborar, tornando possível a coleta de dados.

\section{Sobre as autoras:}

Eliane de Sousa Furtado: Graduada em Psicologia pela UERJ.

Eliane Mary de Oliveira Falcone: Doutora em Psicologia pela USP; professora adjunta do Instituto de Psicologia da UERJ; professora do programa de pós-graduação em Psicologia Social da UERJ.

Cynthia Clark: Doutora em Psicologia pela USP; professora adjunta do Instituto de Psicologia da UERJ e da UFRJ; professora do programa de pós-graduação em Psicologia da UFRJ.

Endereço para correspondência: Rua Paquequer 224/908 Abolição Rio de Janeiro RJ CEP: 20751-300 - E-mail: efurtado@uerj.br 
